\title{
PRIMER REGISTRO DEL GÉNERO ANOCHETUS MAYR 1861 (HYMENOPTERA: FORMICIDAE) PARA EL ESTADO DE JALISCO, MÉXICO
}

Vásquez-Bolaños, M. \& A. Meza-López. 2011. First record of ant genus Anochetus Mayr 1861 (Hymenoptera: Formicidae) from Jalisco state, Mexico. Acta Zoológica Mexicana (n. s.), 27(3): 887-889.

ABSTRACT. The genus Anochetus Mayr is recorded by first time from Jalisco State, and it was known just in the middle and southeastern states in México. It was collected at $9 \mathrm{~km}$ to South to the urban zone in Puerto Vallarta. The specimen revised belongs to species A. mayri Emery. The distribution of the genus and the species is increased to the occidental area in the county.

El género Anochetus Mayr, 1861 pertenece a la tribu Ponerini Lepeletier, 1835 dentro de la subfamilia Ponerinae Lepeletier, 1835. Son hormigas que no rebasan los $5 \mathrm{~mm}$ de longitud total, de hábitos depredadores especialistas, se distinguen de otros géneros por las mandíbulas largas y rectas con dos dientes en el ápice, además el margen posterior de la cabeza termina en $\mathrm{U}$, carácter que permite diferenciarlo del género Odontomachus Latreille, que además es de mayor tamaño, de 6 a $9 \mathrm{~mm}$. Cuenta con 101 especies (incluyendo fósiles) distribuidas en los trópicos de todo el planeta, para el continente Americano se conocen 31 especies restringidas a la región Neotropical. Excepto A. mayri Emery que se distribuye hasta la región Neártica, en Florida, considerada introducida en los Estados Unidos de Norteamérica (Agosti \& Johnson 2005; Bolton et al. 2006; Brown 1978).

En México, se han registrado cuatro especies: A. emarginatus (Fabricius 1804) la cual se conoce para el estado de Quintana Roo (Dejean et al. 1995; RodríguezGarza \& Prisco-Pastrana 2008); A. mayri Emery 1884, conocida para los estados de Morelos (Quiroz-Robledo \& Valenzuela-González 2007), Quintana Roo (RodríguezGarza \& Prisco-Pastrana 2008), Tabasco (Del Toro et al. 2009), Veracruz (Brandão 1991) y Yucatán (Rodríguez-Garza 2008); A. micans conocida para los estados de

Recibido: 11/05/2011; aceptado: 05/07/2011. 
Chiapas y Quintana Roo (García et al. 2003) y A. orchidicola Brown, 1978 conocida para el estado de Veracruz (Brandão 1991).

Se colectó un ejemplar a nueve kilómetros al sur de la ciudad de Puerto Vallarta, Jalisco, por medio de una trampa de caída cebada con miel de maple, cerca de la costa del océano Pacífico casi a nivel del mar. El sitio cuenta con vegetación de bosque tropical subcaducifolio y en sus proximidades se encuentran desarrollos turísticos. Material examinado: Una obrera, etiquetada con los siguientes datos: México, Jalisco, Puerto Vallarta, Garza Blanca, trampa de caída: maple, BTSC, 20 33' 6" N 105 16' 0.9" O, 5-8.XII.2008, 29m., A. Meza-López col. El ejemplar se encuentra depositado en la Sección Formicidae de la Colección Entomológica del Centro de Estudios en Zoología de la Universidad de Guadalajara (CZUG).

El ejemplar revisado pertenece a la especie A. mayri mide $2.5 \mathrm{~mm}$ de longitud total, el pecíolo tiene dos pequeñas espinas en el ápice. Esta especie presenta la distribución más amplia México, se encuentra presente en cinco estados del centro y sureste del país. Hasta el momento, no se había registrado ninguna especie del género Anochetus para el Estado de Jalisco (Vásquez-Bolaños \& Navarrete-Heredia 2004), por lo que representa el primer registro de este género para el estado. Con esta información, se amplia la distribución del género hacia el occidente del país, representando el punto más noroccidental en su distribución original dentro de la región neotropical.

\section{LITERATURA CITADA}

Agosti, D. \& N. F. Johnson (Eds.). 2005. Antbase. World Wide Web electronic publication. antbase. org.

Bolton, B., G. Alpert, P. S. Ward \& P. Naskrecki. 2006. Bolton's catalogue of ants of the world: 1758-2005. Harvard University Press, Cambridge Massachusetts. CDroom.

Brandão, C. R. F. 1991. Adendos ao catálogo abreviado das formigas da regiâo neotropical (Hymenoptera: Formicidae). Revista Brasileira de Entomologia, 35: 319-412.

Brown, W. L. Jr. 1978. Contribution toward a reclassification of the Formicidae. Part VI Ponerinae, Tribe Ponirini, Subtribe Odontomachiti. Section B. Genus Anochetus and bibliography. Studia Entomological, 20: 549-652.

Dejean, A., Olmsted \& R. R. Snelling. 1995. Trees-epiphytes-ants relationships in the biosphera reserve of Sian Ka'an, Quintana Roo, Mexico. Biotropica, 27: 57-70.

Del Toro, I., M. Vásquez, W. P. Mackay, P. Rojas \& R. Zapata-Mata. 2009. Hormigas (Hymenoptera: Formicidae) de Tabasco: explorando la diversidad de la mirmecofauna en las selvas tropicales de baja altitud. Dugesiana, 16: 1-14.

García, M. D., R. W. Jones, W. P. Mackay \& P. Rojas. 2003. Diversity and habitat associations of the ants (Insecta: Formicidae) of El Edén Ecological Reserve, pp. 293-304. In: Gómez-Pompa, A., M. F. Allen, S. L. Fedick \& J. J. Jimenez-Osornio (Eds.). The lowland Maya area, three millennia at the human-wildland interface. Food Products Press (The Haworth Press), New York.

Quiroz-Robledo, L. N. \& J. Valenzuela-González. 2007. Distribution of poneromorph ants (Hymenoptera: Formicidae) in the Mexican state of Morelos. Florida Entomologist, 90: 609-615.

Rodríguez-Garza, J. A. 2008. Hormigas (Hymenoptera: Formicidae) del estado de Yucatán: nuevos registros. Entomología Mexicana, 7: 1006-1008. 
Rodríguez-Garza, J. A. \& J. A. Prisco-Pastrana. 2008. Hormigas de los géneros Anochetus y Odontomachus (Hymenoptera: Formicidae) recolectados en el sur de Quintana Roo. Entomología Mexicana, 7: 1009-1010.

Vásquez-Bolaños, M. \& J. L. Navarrete-Heredia. 2004. Checklist of the ants (Hymenoptera: Formicidae) from Jalisco state, Mexico. Sociobiology, 43: 351-365.

\section{Miguel VÁsQuez-Bolaños* \& Abraham Meza-LóPEZ}

Entomología, Centro de Estudios en Zoología,

Centro Universitario de Ciencias Biológicas y Agropecuarias,

Universidad de Guadalajara,

Apdo. Postal 134, Zapopan, Jalisco,

C.P. 45100, MÉXICO

*<mvb14145@hotmail.com> 